\title{
Effect of Chelate Type and Radioisotope on the Imaging Efficacy of 4 Fibrin-Specific PET Probes
}

\author{
Francesco Blasi*, Bruno L. Oliveira*, Tyson A. Rietz, Nicholas J. Rotile, Helen Day, Richard J. Looby, Ilknur Ay, \\ and Peter Caravan \\ Athinoula A. Martinos Center for Biomedical Imaging, Department of Radiology, Massachusetts General Hospital, Charlestown, \\ Massachusetts
}

Thrombus formation plays a major role in cardiovascular diseases, but noninvasive thrombus imaging is still challenging. Fibrin is a major component of both arterial and venous thrombi and represents an ideal candidate for imaging of thrombosis. Recently, we showed that ${ }^{64} \mathrm{Cu}$-DOTA-labeled PET probes based on fibrin-specific peptides are suitable for thrombus imaging in vivo; however, the metabolic stability of these probes was limited. Here, we describe 4 new probes using either ${ }^{64} \mathrm{Cu}$ or aluminum fluoride $\left(\mathrm{Al}{ }^{18} \mathrm{~F}\right)$ chelated to 2 NOTA derivatives. Methods: Probes were synthesized using a known fibrin-specific peptide conjugated to either NODAGA (FBP8, FBP10) or NOTA-monoamide (FBP9, FBP11) as chelators, followed by labeling with ${ }^{64} \mathrm{Cu}$ (FBP8 and FBP9) or $\mathrm{Al}^{18} \mathrm{~F}$ (FBP10 and FBP11). PET imaging efficacy, pharmacokinetics, biodistribution, and metabolic stability were assessed in a rat model of arterial thrombosis. Results: All probes had similar nanomolar affinity (435-760 $\mathrm{nM}$ ) for the soluble fibrin fragment $\mathrm{DD}(\mathrm{E})$. PET imaging allowed clear visualization of thrombus by all probes, with a 5-fold or higher thrombus-to-background ratio. Compared with the previous DOTA derivative, the new ${ }^{64} \mathrm{Cu}$ probes FBP8 and FBP9 showed substantially improved metabolic stability $(>85 \%$ intact in blood at $4 \mathrm{~h}$ after injection), resulting in high uptake at the target site $(0.5-0.8$ percentage injected dose per gram) that persisted over $5 \mathrm{~h}$, producing increasingly greater target-to-background ratios. The thrombus uptake was 5- to 20-fold higher than the uptake in the contralateral artery, blood, muscle, lungs, bone, spleen, large intestine, and heart at $2 \mathrm{~h}$ after injection and 10- to 40 -fold higher at $5 \mathrm{~h}$. The $\mathrm{Al}^{18} \mathrm{~F}$ derivatives FBP10 and FBP11 were less stable, in particular the NODAGA conjugate (FBP10, $<30 \%$ intact in blood at $4 \mathrm{~h}$ after injection), which showed high bone uptake and low thrombus-tobackground ratios that decreased over time. The high thrombus-to-contralateral ratios for all probes were confirmed by ex vivo biodistribution and autoradiography. The uptake in the liver $(<0.5$ percentage injected dose per gram), kidneys, and blood were similar for all tracers, and they all showed predominant renal clearance. Conclusion: FBP8, FBP9, and FBP11 showed excellent metabolic stability and high thrombus-to-background ratios and represent promising candidates for imaging of thrombosis in vivo.

Received Dec. 27, 2013; revision accepted Mar. 14, 2014.

For correspondence or reprints contact: Peter Caravan, Building 149, Rm. 2301, 13th St., Charlestown, MA 02129.

E-mail: caravan@nmr.mgh.harvard.edu

${ }^{*}$ Contributed equally to this work.

Published online May 1, 2014.

COPYRIGHT (c) 2014 by the Society of Nuclear Medicine and Molecular Imaging, Inc.
Key Words: ${ }^{64} \mathrm{Cu}$; $\mathrm{Al}{ }^{18} \mathrm{~F}$; positron emission tomography; fibrin; thrombosis

J Nucl Med 2014; 55:1157-1163

DOI: 10.2967/jnumed.113.136275

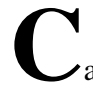

ardiovascular diseases, including heart attack, stroke, deep vein thrombosis, and pulmonary embolism, are leading causes of death and disability worldwide (1). Thrombosis is a common feature and often the underlying cause of most cardiovascular disorders; therefore, the early detection of thrombus formation is critical for both diagnosis and intervention. Current imaging modalities for thrombus detection can be somewhat invasive (e.g., transesophageal echocardiography (2)) and do not offer a single approach with high sensitivity and specificity to detect thrombosis in different vascular territories; rather multiple techniques specific for distinct territories are used (e.g., transesophageal echocardiography for atrial thrombus (2), ultrasonography for pelvic and carotid thrombosis (3), and CT for pulmonary embolism (4)). Direct targeting of thrombus components using molecular imaging approaches offers instead a noninvasive solution with high sensitivity and potential full-body application. Many components of the coagulation system have been targeted to image thrombosis, including Factor XIII (5), activated platelets (6), and fibrinogen and fibrin $(7,8)$. Among these, fibrin represents a potentially ideal target for molecular imaging of thrombosis (9): it is present at high concentration in all thrombi (arterial and venous, fresh and aged), resulting in potentially high sensitivity, but is absent in circulating blood, suggesting high specificity. In the last decade, fibrin-specific MR probes showed high feasibility for thrombus imaging in both preclinical research $(7,10)$ and clinical trials $(11)$. Building on our previous results using the fibrin-targeted MR contrast agent EP-2104R, we recently developed PET/MR and PET-only probes for molecular imaging of thrombosis $(12,13)$. We conjugated ${ }^{64} \mathrm{Cu}-1,4,7,10$-tetraazacyclododecane-1,4,7,10-tetraacetic acid ( ${ }^{64} \mathrm{Cu}$-DOTA) to 3 fibrin-specific peptides and assessed imaging properties, uptake, and stability of these probes in a rat model of carotid thrombosis (13). However, the metabolic stability of these probes was limited by some dissociation of ${ }^{64} \mathrm{Cu}$ from the DOTA chelator, resulting in persistent blood background. To address the issue of copper dissociation, here we synthesized 4 new fibrin-binding probes (FBPs) by replacing DOTA with either the 1,4,7-triazacyclononane,1-glutaric acid-4,7-acetic acid (NODAGA) or the 1,4,7-triazacyclononane-1,4,7-triacetic acid (NOTA)-monoamide chelator, both known to form stable complexes with copper (14). 
These NOTA derivatives can also be labeled with ${ }^{18} \mathrm{~F}$ via complexation of aluminum fluoride $\left(\mathrm{Al}{ }^{18} \mathrm{~F}\right)(15)$. The goal of this study was to evaluate these 4 new FBPs and to directly compare how these different chelators and radioisotopes affect target uptake, imaging efficacy, pharmacokinetic properties, and metabolic stability using a rat model of arterial thrombosis.

\section{MATERIALS AND METHODS}

Additional information is reported in the supplemental material (available at http://jnm.snmjournals.org).

\section{Precursor and Cold Probe Syntheses}

The cyclic disulfide peptide precursor FHCHypY(3-Cl)DLCHILPXD (Hyp $=L$-4-hydroxyproline, $\mathrm{Y}(3-\mathrm{Cl})=L$-3-chlorotyrosine, PXD = para-xylenediamine) was prepared using the solid-phase peptide synthesis from a xylenediamine trityl resin. The general synthetic route of FBP8, FBP9, FBP10, and FBP11 is depicted in Figure 1. Briefly, the activated ester NODAGA- $N$-hydroxysuccinimide was directly coupled to the $\mathrm{N}$ and $\mathrm{C}$ termini of $\mathrm{FHCHypY(3-Cl)DLCHIL-PXD}$ (Pep), affording the precursor (NODAGA) ${ }_{2}$ Pep. The precursor (NOTAmonoamide) $)_{2}$ Pep was prepared by coupling di-tbutyl-protected NOTA as the pentafluorophenol ester to the cyclic peptide, followed by hydrolysis of the tbutyl-protecting groups. Reaction of (NODAGA) ${ }_{2} \mathrm{Pep}$ and (NOTA-monoamide) $)_{2}$ Pep with an excess of either $\mathrm{CuSO}_{4}$ or $\mathrm{AlCl}_{3}$ and $\mathrm{NaF}$ resulted in the synthesis of the nonradioactive surrogates FBP8 $(\mathrm{Cu})$, FBP9 $(\mathrm{Cu})$, FBP10 (AlF), and FBP11 $(\mathrm{AlF})$. All intermediates and final compounds were purified by high-performance liquid chromatography (HPLC) and characterized by liquid chromatography-mass spectrometry. The chemical purities of these compounds were greater than 97\%, determined by analytic HPLC analysis.

\section{Synthesis, In Vitro Stability, and Affinity of ${ }^{64} \mathrm{Cu}$ - and} Al ${ }^{18}$ F-Labeled Probes

A $50-\mu \mathrm{L}$ aliquot of a $1 \mathrm{mg} / \mathrm{mL}$ solution of either (NODAGA) ${ }_{2} \mathrm{Pep}$ or (NOTA-monoamide) ${ }_{2}$ Pep $(23 \mathrm{nmol})$ was added to ${ }^{64} \mathrm{CuCl}_{2}(3.7 \times$ $10^{9} \mathrm{~Bq}[10 \mathrm{mCi}]$ ), diluted in $400 \mu \mathrm{L}$ of NaOAc, pH 5.5, and heated at $50^{\circ} \mathrm{C}$ for $30 \mathrm{~min}$. No further purification was required. Fluorination of the fibrin-biding peptides was based on the chelation of the $\left[\mathrm{Al}^{18} \mathrm{~F}\right]^{2+}$ com-

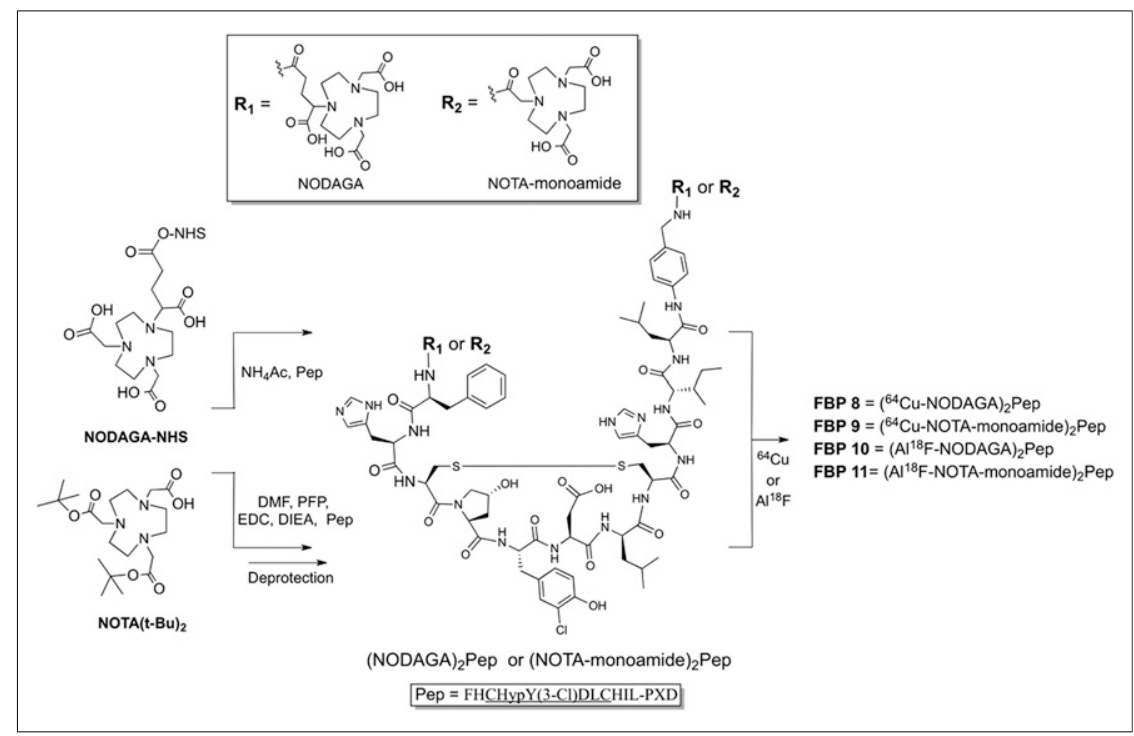

FIGURE 1. General protocol for synthesis of the fibrin-binding PET probes (FBPs). plex by NODAGA or NOTA-monoamide as described elsewhere (15). Briefly, a QMA cartridge (Sep-Pak; Waters) was used to concentrate ${ }^{18} \mathrm{~F}^{-}$, which was eluted from the column with $\mathrm{KHCO}_{3}$ and acidified to $\mathrm{pH} 4.1$ with acetic acid. Then, $15 \mu \mathrm{L}$ of a $2.5-\mathrm{mM}$ solution of (NODAGA) $)_{2} \mathrm{Pep}$ or (NOTA-monoamide) $)_{2}$ Pep $(37.5 \mathrm{nmol})$ were mixed with $6 \mathrm{nmol}$ of $\mathrm{AlCl}_{3}$ and approximately $9.25 \times 10^{8} \mathrm{~Bq}(25 \mathrm{mCi})$ of ${ }^{18} \mathrm{~F}^{-}$, heated for $15 \mathrm{~min}$ at $104^{\circ} \mathrm{C}$, and purified by Sep-Pak cartridge (Waters). Radiolabeling yields and radiochemical purities were monitored by analytic HPLC.

Stability in bovine serum was assessed by radio-HPLC analysis after incubation of the probes at $37^{\circ} \mathrm{C}(\leq 18 \mathrm{~h}$ for copper derivatives FBP8 and FBP9 and $\leq 3 \mathrm{~h}$ for the AlF derivatives FBP10 and FBP11). Transchelation experiments for FBP10 and FBP11 were performed by incubating the probes with an excess of NOTA for up to $30 \mathrm{~min}$ $\left(37^{\circ} \mathrm{C}\right.$ and $104^{\circ} \mathrm{C}$ ), followed by radio-HPLC analysis (Supplemental Table 3).

Fibrin affinity of the nonradioactive surrogates was assessed using an established fluorescence polarization assay by measuring the displacement of a fluorescent peptide bound to the soluble fibrin fragment DD (E) as a function of added FBP (13).

\section{Animal Model and Probe Administration}

All experiments were performed in accordance with the National Institutes of Health Guide for the Care and Use of Laboratory Animals (16) and were approved by the Institutional Animal Care and Use Committee at Massachusetts General Hospital. Adult male Wistar rats ( $n=37$; weight, 330-360 g; Charles River Laboratories) were used for this study. Arterial thrombosis was induced by carotid crush injury. Under isoflurane anesthesia, the right common carotid artery was exposed, and a deep external crush injury was induced by clamping the vessel for $5 \mathrm{~min}$, as previously described (13). Injury was performed 1-2 cm proximal to carotid bifurcation, using the same hemostat and by the same investigator to minimize variability. The femoral vein and artery were catheterized using PE-50 tubing (Fisher Scientific) for probe injection and blood sampling, respectively. Probes were injected 15-30 min after thrombus formation. Each rat was injected with approximately $1.11 \times 10^{8} \mathrm{~Bq}(300 \mu \mathrm{Ci})$ in approximately $0.4 \mathrm{~mL}$ of the dose solution measured with a dose calibrator (CRC-25PET; Capintec). This relatively high radiochemical dose was needed to ensure that there was measurable activity in the clot and contralateral vessel, both of which weighed less than $10 \mathrm{mg}$.

\section{PET Imaging and Analysis}

PET scans were obtained with a dedicated small-animal PET/SPECT/CT scanner (Triumph; TriFoil Imaging), equipped with gas anesthesia and heating system. Instrument calibration was performed each day by scanning a phantom of known activity. After carotid crush induction, rats were transferred into the scanner and imaged either for 90 min immediately after the injection of the probe or for $45 \mathrm{~min}$ at $240 \mathrm{~min}$ after injection. The PET field of view was $80 \mathrm{~mm}$ and covered the head to the base of the heart with the neck at the isocenter. After the PET acquisition, a CT scan was obtained with a constant infusion of iodinated contrast (iopamidol; Isovue 370 [Bracco], $0.3 \mathrm{~mL} / \mathrm{min}$ ). Images were acquired over 6 min with 512 projections with 3 frames per projection (exposure time per frame, $\sim 200$ $\mathrm{ms}$; peak tube voltage, $70 \mathrm{kV}$; tube current, 177 $\mathrm{mA}$ ). Animals were euthanized at the end 
of the imaging session and tissues were harvested and processed for biodistribution, autoradiography, metabolic stability, and functional fibrin-binding assay.

PET and CT images were reconstructed using the LabPET software (TriFoil Imaging) to a voxel size of $0.5 \times 0.5 \times 0.6 \mathrm{~mm}$ (PET) and isotropic $0.3 \mathrm{~mm}^{3}$ (CT). Data obtained from the $90-\mathrm{min}$ scan were framed into a dynamic sequence of $10 \times 60,10 \times 180$, and $5 \times 600 \mathrm{~s}$; an additional image was reconstructed from the 45 -min scan. Data of each frame were reconstructed using an iterative algorithm (maximum-likelihood expectation maximization, 30 iterations). All images were corrected for decay, randoms, and dead time; CT data were used to provide for attenuation correction. Reconstructed PET/CT data were quantitatively evaluated using AMIDE (17) and PMOD 3.2 (PMOD Technologies Ltd.) software packages. Volumes of interest were drawn using fused, coregistered CT and PET images to localize the hot spot at the site of the injured common carotid artery. Volumes of interest were also drawn in the brain, bone (spinous process of cervical vertebrae), muscle (acromiotrapezius), heart, and contralateral artery. Results were expressed as percentage injected dose per cubic centimeter of tissue.

\section{Ex Vivo Studies}

Serial blood samples were collected in ethylenediaminetetraacetic acid tubes and the radioactivity measured on a $\gamma$ counter (CobraII Auto-Gamma; Packard) to assess clearance of total activity. To measure the amount of functional probe, plasma samples were checked for fibrin binding by incubation with immobilized fibrin in a microtiter plate. To evaluate in vivo stability, blood plasma samples at 2, 15, 120, and 240 min were filtered and injected onto an analytic HPLC column. The eluent was collected every $30 \mathrm{~s}$, and the activity of each fraction was measured by a $\gamma$ counter. Probe half-lives were calculated from a biexponential fit to the clearance data of the functional probes.

For the biodistribution studies, the animals were euthanized 2 or $5 \mathrm{~h}$ after injection, and the distributions of ${ }^{64} \mathrm{Cu}$ - and $\mathrm{Al}^{18} \mathrm{~F}$-labeled probes in the thrombus, contralateral left carotid artery, blood, internal organs, brain, left rectus femoris muscle, and left femur bone were quantified. The tissues were weighed, and radioactivity in each tissue was measured and compared with an aliquot of the injected dose solution, to determine the percentage injected dose per gram of tissue $(\% \mathrm{ID} / \mathrm{g})$. Right and left carotid arteries were further analyzed by autoradiography using a multipurpose film and a PerkinElmer Cyclone Plus Storage Phosphor system and quantified using PerkinElmer OptiQuant 5.0 software. Regions of interest were drawn around ipsilateral (injured) and contralateral carotid arteries to obtain raw values expressed as digital light units $/ \mathrm{mm}^{2}$. Ipsilateral-to-contralateral activity ratios were obtained by dividing matched ipsilateral and contralateral raw values from each animal.

\section{Statistics}

Data were expressed as mean \pm SEM. Differences between groups were compared using the Student $t$ test and ANOVA, followed by the
Bonferroni or Dunnett post hoc test, as appropriate. A $P$ value of less than 0.05 was considered significant.

\section{RESULTS}

\section{Synthesis, In Vitro Stability, and Affinity of FBPs}

The general synthetic route for the probes is depicted in Figure 1. FBP8 and FBP9 were obtained by reaction of the ligands with ${ }^{64} \mathrm{CuCl}_{2}$ in yields of $99 \%$ or greater and with specific activities of $0.21-0.43 \mathrm{mCi} / \mathrm{nmol}$. In optimized conditions, FBP10 and FBP11 were obtained with yields of $57 \% \pm 6 \%$ and $81 \% \pm 5 \%$, respectively (Supplemental Tables 1 and 2). Considerable improvements in radiochemical yield were obtained when the labeling was performed in the presence of an organic solvent. Pure FBP10 and FBP11 $(\geq 97 \%)$ were obtained after Sep-Pak purification with specific activities of $0.30-0.36 \mathrm{mCi} / \mathrm{nmol}$. The nonradioactive surrogates were obtained by reaction of the ligands with an excess of metal ion, followed by HPLC purification (purity $\geq 98 \%$ ).

All probes showed high stability in serum $(>99 \%)$. NOTA challenge experiments revealed similar stability for FBP10 and FBP11 after incubation at $37^{\circ} \mathrm{C}$; however, FBP11 was less susceptible to transchelation than FBP10 when incubated at $104^{\circ} \mathrm{C}$ (Supplemental Table 3). Competitive binding studies showed that all probes displayed similar affinity to fibrin (435-760 nM, Supplemental Table 4), comparable to that of the MR probe EP-2104R.

\section{PET Imaging}

Radiolabeled probes were investigated in a rat model of arterial thrombosis. The presence of mural thrombi was verified by histology (Supplemental Fig. 5). Representative CT, PET, and fused PET/CT images, summed over 30-90 min after administration of FBP8, are shown in Figure 2. The thrombus was visualized as a region of high activity in PET. Fused images show that the hyperintense PET signal corresponds to the level of the common carotid artery as visualized with CT angiography. An additional area of high activity in PET was seen superficially at the surgical site, most likely due to FBP8 binding to clotted blood at the wound site $(10,13)$. The wound uptake did not affect PET quantification at the level of the carotid arteries (Supplemental Fig. 6).

The direct comparison of the imaging properties and region-ofinterest analyses for all 4 probes at 30-90 min after injection is shown in Figure 3. All the probes were able to clearly detect the thrombus from background, with minimal nontarget uptake. For both copper probes (FBP8 and FBP9), the thrombus was easily identified as a hot spot, with high contrast between the target and the background tissue and organs. Time-activity curves showed a steady level of probe uptake at the target thrombus but fast washout from nontarget organs. Figure 3B shows the averaged time-activity

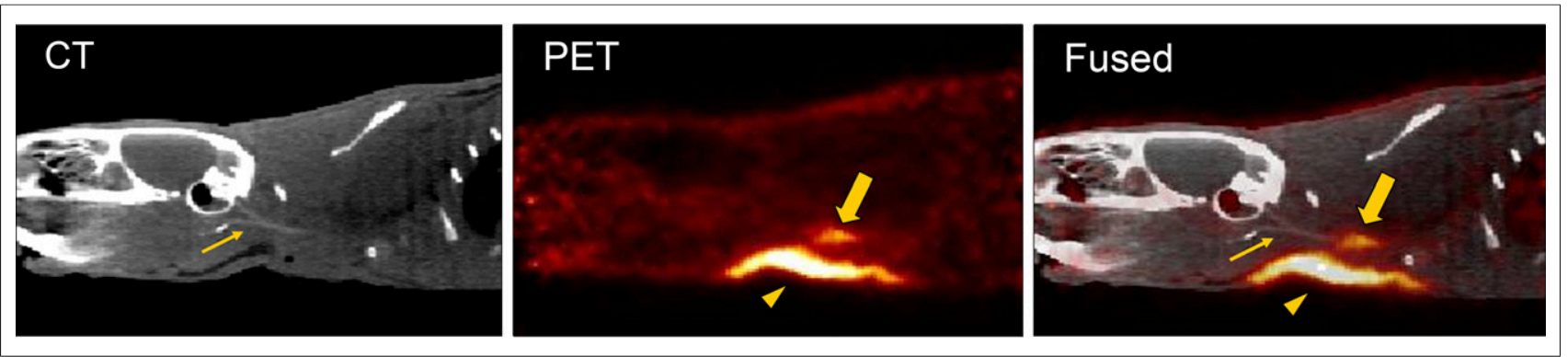

FIGURE 2. Representative sagittal views of CT, PET, and fused PET/CT images reconstructed from data $30-90$ min after injection of FBP8. Arrow indicates thrombus; arrowhead, surgical area; and thin arrow, common carotid artery visualized by CT angiography. 
curves in the thrombus and contralateral carotid for the 4 probes. Additional time-activity curves for bone, heart, and muscle are presented in Supplemental Figure 2. One-way ANOVA between activity values for thrombus versus background tissues revealed strong statistical differences (Fig. 3C, $P<0.0001$ ). AlF probes (FBP10 and FBP11) were also able to detect the thrombus but with lower target-to-background ratios. Off-target signal in the bone was evident, especially for FBP10. This effect was probably due to the partial dissociation of ${ }^{18} \mathrm{~F}$-fluoride from the chelate. For FBP11, the activity in the thrombus was greater than in the contralateral artery, heart, bone, muscle, or brain (Fig. 3C, $P<0.0001$ ). Statistical analysis for FBP10 showed no significant differences between target and background organs (Fig. 3C). At the later time point (240-285 min after injection, Supplemental Figs. 1A-1H), the copper probes allowed a better visualization of the clot than the fluorine probes. $\mathrm{Al}^{18} \mathrm{~F}$-labeled tracers, especially $\mathrm{FBP} 10$, showed substantive bone accumulation, suggesting in vivo defluorination.

The retention of activity in the thrombus and the time-dependent reduction of the off-target activity results in an increased thrombusto-background ratio with time. In particular, all the probes showed a 4- to 10-fold contrast between the thrombus and the contralateral artery, heart, and muscle at 30-90 min after injection (Supplemental Fig. 3A). In the case of the copper probes FBP8 and FBP9, continued off-target washout resulted in an increased thrombus conspi- cuity with thrombus-to-background ratios greater than 20:1 (240-285 min after injection, Supplemental Fig. 3B). Differently, fluorine derivatives FBP10 and FBP11 did not benefit from delayed imaging.

\section{Ex Vivo Studies}

Biodistribution studies confirmed the high uptake of all the probes in the thrombus (Fig. 4). At 120 min after injection, the thrombus was the tissue with the second highest uptake $(0.6-0.8 \% \mathrm{ID} / \mathrm{g})$, just after the kidneys, except for FBP10, which also showed high bone activity $(1.5 \% \mathrm{ID} / \mathrm{g})$. For all the probes, there was 3-6 times more activity in the ipsilateral thrombotic artery than in the contralateral vessel. Notably, liver uptake was approximately 50\% lower than the thrombus for both copper probes and for FBP10; FBP11 showed comparable values instead. Very low uptake was detected for the muscle, brain, and blood, as well as for the chest and abdominal organs, except for FBP11, which slightly accumulated in the spleen $(\sim 0.2 \% \mathrm{ID} / \mathrm{g})$ and intestine $(\sim 0.5 \% \mathrm{ID} / \mathrm{g})$. For all the probes, the thrombus had more than 10-fold-higher activity than heart, lungs, muscle, brain, and blood (Supplemental Fig. 4A).

At $300 \mathrm{~min}$ after injection, the thrombus uptake $(\sim 0.4 \% \mathrm{ID} / \mathrm{g})$ was $40 \%-50 \%$ lower than at $120 \mathrm{~min}$ but still much higher than the background (Fig. 4C). Besides kidney and liver, and bone in the case of FBP10, the uptake in the other organs and tissues was extremely low $(<0.1 \% \mathrm{ID} / \mathrm{g})$. This low uptake resulted in high

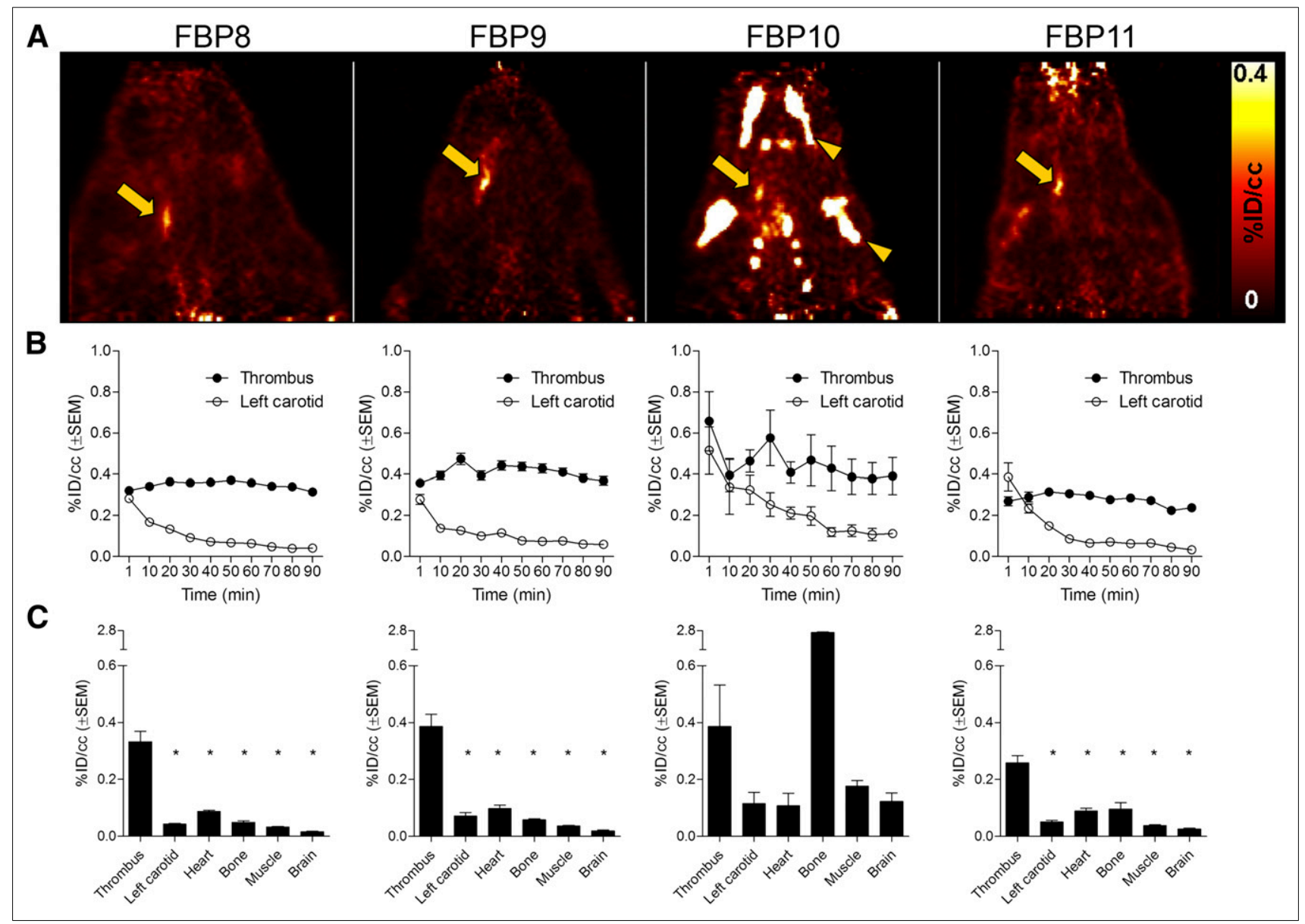

FIGURE 3. Side-by-side comparison of PET images (A) after injection of FBP8 $(n=7)$, FBP9 $(n=5)$, FBP10 $(n=3)$, and FBP11 ( $n=5)$; correspondent time-activity curves (B) of thrombus and left carotid obtained from dynamic PET imaging; correspondent mean PET activity values (C) of thrombus, left carotid, heart, bone, muscle, and brain (30-90 min after injection). Bone activity for FBP10 is off scale. Arrow indicates thrombus, and arrowhead indicates bone uptake. ${ }^{\star} P<0.0001$, vs. thrombus. 


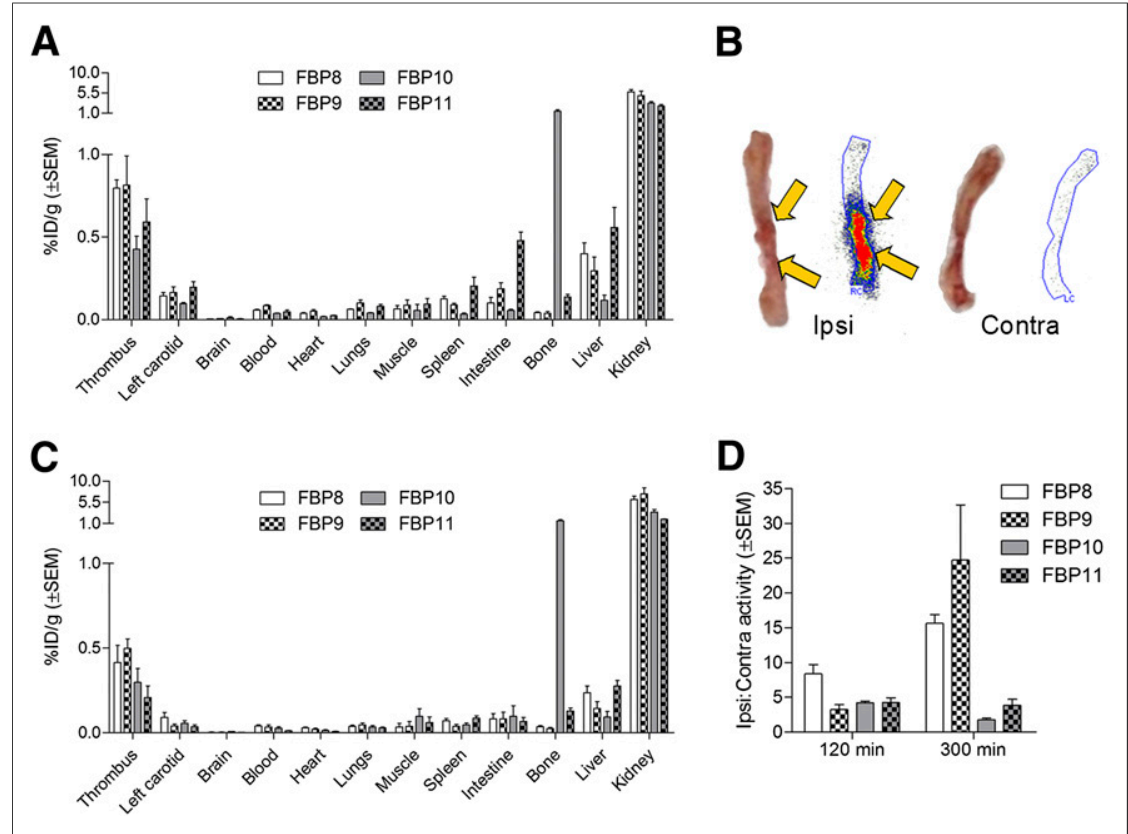

FIGURE 4. Biodistribution for FBP8, FBP9, FBP10, and FBP11 at $120 \mathrm{~min}$ (A, $n=3-7 /$ probe) and $300 \mathrm{~min}$ (C, $n=3-5 /$ probe) after injection. Representative photograph and autoradiogram of ipsilateral and contralateral carotid arteries taken from a rat at $120 \mathrm{~min}$ after FBP8 injection (B). Mean activity ratios from autoradiography for each probe at 120 and $300 \mathrm{~min}$ after injection ( $\mathrm{D}, n=3-6 /$ probe).

Ex vivo autoradiography confirmed the results obtained from PET imaging and biodistribution. A hyperintense region was detected in the right carotid segment corresponding to the location in which the artery was crushed but not in the contralateral vessel (Fig. 4B). As shown in Figure 4D, at $120 \mathrm{~min}$ after injection there was a 4- to 8-fold difference between right and left carotid with all the probes, and this difference increased to 15- to 20-fold at the later time point for FBP8 and FBP9. For the $\mathrm{Al}^{18} \mathrm{~F}$ probes FBP10 and FBP11, there was no enhancement of target to background at the later time point.

Serial blood draws were collected up to 120 min after injection to determine the clearance of activity from the blood, to assess the fraction of functional FBP, and to calculate blood half-lives. All probes showed a similar and rapid elimination from circulation, congruent with the low background activity in biodistribution and imaging studies (Fig. 5). The results from the fibrin-binding assay showed that the concentration of functional FBP8, FBP9, and FBP11 was similar to the total concen-

thrombus-to-background ratios (Supplemental Fig. 4B), especially in the heart (15- to 30-fold), blood (10- to 20-fold), and muscle (30- to 40-fold) for the copper probes and 10- to 20-fold for the fluorine probes. A complete list of biodistribution values for all 4 probes is reported in Supplemental Tables 5 and 6.
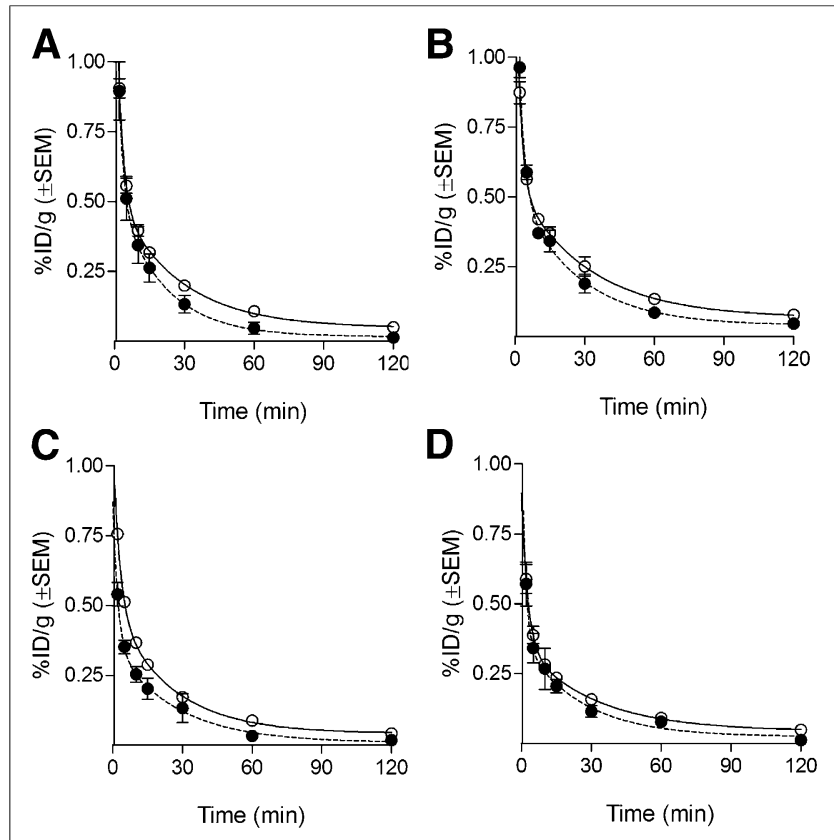

FIGURE 5. Pharmacokinetic data from ex vivo blood analyses for FBP8 (A, $n=7)$, FBP9 (B, $n=5)$, FBP10 (C, $n=3)$, and FBP11 (D, $n=5)$. $\mathrm{O}=$ total ${ }^{64} \mathrm{Cu} /{ }^{18} \mathrm{~F}$ activity in blood; $\boldsymbol{0}=$ functional $\mathrm{FBP}$ (intact). tration of activity in the blood, suggesting a high in vivo metabolic stability for these 3 probes. We estimated elimination half-lives for the intact probes of $13.8 \pm 3.8 \mathrm{~min}(\mathrm{FBP} 8), 17.9 \pm 5.8 \mathrm{~min}$ (FBP9), $18.4 \pm 10.0 \mathrm{~min}(\mathrm{FBP} 10)$, and $17.3 \pm 12.5 \mathrm{~min}$ (FBP11).

HPLC of blood plasma confirmed the functional assay data (Fig. 6). FBP8 and FBP9 were largely intact $(>85 \%)$ over the entire study. FBP10 ( $\mathrm{Al}^{18} \mathrm{~F}$ chelated by NODAGA) was the least stable probe, showing 20\% decomposition even at $2 \mathrm{~min}$ after injection. HPLC traces for FBP10 showed a peak corresponding to intact probe and a peak with a retention time of free fluoride, supporting the hypothesis that the high bone uptake of this probe is due to in vivo defluorination. The $\mathrm{Al}^{18} \mathrm{~F}-\mathrm{NOTA}$-monoamide $\mathrm{FBP} 11$ was more stable $(>70 \%$ intact).

\section{DISCUSSION}

The goal of this study was to perform a head-to-head comparison of target uptake, imaging properties, pharmacokinetics, and metabolic stability of 4 new fibrin-specific PET probes. We recently reported the efficacy of a ${ }^{64} \mathrm{Cu}$-DOTA-labeled peptide, FBP2, for molecular imaging of thrombosis (13). The strategy of using a small peptide for targeting offers potential advantages of high clot penetration, faster blood clearance, and lower cost of production when compared with nanoparticle- or antibody-based approaches (18). With FBP2, we noted partial release of ${ }^{64} \mathrm{Cu}$ from the DOTA chelator, resulting in elevated background signal (13). We reasoned that replacing DOTA with NOTA would limit ${ }^{64} \mathrm{Cu}$ release and result in increased target-to-background ratio. It was not obvious which NOTA chelator would perform better, so we tested NODAGA (anionic copper complex, 3 carboxylate donors) and NOTAmonoamide (neutral complex, 2 carboxylate donors). We were also interested in directly comparing the ${ }^{64} \mathrm{Cu}$ probes with 


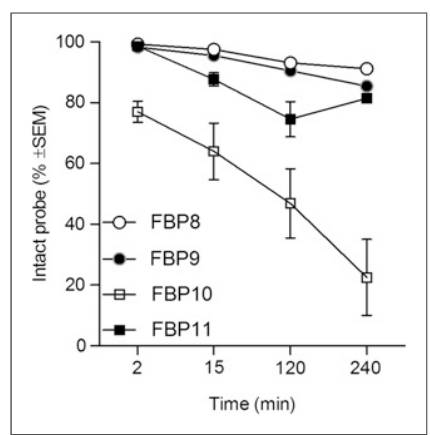

FIGURE 6. Metabolic stability of each probe estimated from HPLC analysis of blood samples $(n=2$ / probe).
$\mathrm{A} 1^{18} \mathrm{~F}$-labeled probes and in assessing which chelator performed better with $\mathrm{Al}^{18} \mathrm{~F}$.

All probes showed similar fibrin affinity and good stability when incubated in plasma. All 4 probes were obtained with high radiochemical purity and without the need for HPLC purification. The copper complexes FBP8 and FBP9 were radiolabeled in quantitative yields under mild conditions (30 $\min$ at $50^{\circ} \mathrm{C}$ ). $\mathrm{FBP} 11$ (Al ${ }^{18} \mathrm{~F}$-NOTA-monoamide) consistently showed higher labeling efficiency than $\mathrm{FBP} 10\left(\mathrm{Al}^{18} \mathrm{~F}\right.$ NODAGA), suggesting that the presence of the third free carboxylate group in NODAGA interferes with the coordination of $\mathrm{Al}^{18} \mathrm{~F}$. These findings are supported by in vitro stability studies with free NOTA showing that FBP11 is less prone to transchelation than FBP10.

PET imaging showed that the thrombus target was clearly visualized by all probes at both time points, with at least a 5-fold thrombus-to-background ratio. Ex vivo autoradiography and biodistribution were highly consistent with imaging data. Pharmacokinetic analysis showed a biexponential, rapid elimination of the probes from the blood (elimination half-life, 15-20 min). Compared with the DOTA derivative FBP2, both ${ }^{64} \mathrm{Cu}-\mathrm{NOTA}$ derivatives displayed improved metabolic stability $(>85 \%$ intact in blood from 0 to $4 \mathrm{~h}$ ), resulting in low blood and liver values and excellent target-to-background ratios that increased with time. For the ${ }^{64} \mathrm{Cu}$ probes $\mathrm{FBP} 8$ and $\mathrm{FBP} 9$, the thrombus was the tissue with the highest uptake just after the kidneys. The $\mathrm{Al}^{18} \mathrm{~F}-$ NOTA-monoamide FBP11 also showed a high target-to-background ratio and good metabolic stability; however, there was no benefit to delayed imaging as in the case with the ${ }^{64} \mathrm{Cu}$ probes. On the other hand, the $\mathrm{Al}{ }^{18} \mathrm{~F}-\mathrm{NODAGA}$ derivative FBP10 showed decomposition in vivo that was consistent with defluorination. $\mathrm{A}{ }^{18} \mathrm{~F}-\mathrm{NODAGA}-$ labeled peptides have also displayed more bone uptake than $\mathrm{Al}^{18} \mathrm{~F}-\mathrm{NOTA}$ in mouse models of prostate cancer $(19,20)$.

The rapid target uptake and retention combined with the fast systemic clearance of FBP8, FBP9, and FBP11 make these probes useful candidates for fibrin imaging in several pathologic conditions. Because fibrin is the predominant thrombus component (21), these probes are well suited to cardiovascular imaging to detect the presence of pulmonary embolism, carotid thrombus, cardiac chamber thrombi, or deep vein thrombosis. Moreover, these probes may be useful for atherosclerosis imaging because increased fibrin deposition is associated with higher plaque vulnerability (22). Furthermore, because fibrin accumulates in tumor stroma and plays a role in growth, invasion, and metastasis, these probes can be also tested for cancer imaging (23).

The ability to generate either ${ }^{18} \mathrm{~F}$ - or ${ }^{64} \mathrm{Cu}$-labeled peptides with similar properties provides versatility of use. We adopted an emergent method for ${ }^{18} \mathrm{~F}$ labeling (15) and optimized the synthetic route to prepare FBP11 in a good yield with no HPLC purification. This method is readily adapted to automated synthesis in any radiopharmacy with access to ${ }^{18} \mathrm{~F}^{-}$water; a lyophilized kit for rapid radiofluorination has been recently tested in clinical settings (24). On the other hand, the long half-life of ${ }^{64} \mathrm{Cu}(\sim 12.7 \mathrm{~h})$ allows synthesis of FBP8 or FBP9 in advance or for these probes to be shipped to remote sites on demand. The rapid renal clearance and low whole-body retention of FBP8 and FBP9 suggest that dosimetry concerns will not limit their clinical translation.

The goal of this work was to vary the chelator and radionuclide to optimize thrombus imaging properties of a fibrin-targeted peptide. Three strong candidates emerged with high thrombus uptake, rapid systemic clearance, and low off-target retention. We hypothesized that these properties, along with the small size of the probes, make them improved candidates for fibrin imaging, compared with other approaches (2-6). Moreover, the higher spatial resolution of clinical PET (vs. SPECT) combined with the ability of CT or MR to localize PET signal within the vascular tree offers great potential for detection of small thrombi. These hypotheses are currently being tested in additional animal models and ultimately in human trials.

\section{CONCLUSION}

In this study, we demonstrated the optimization and successful application of 3 new probes for PET detection of acute thrombus formation in vivo. Any of these probes represents a promising candidate for clinical translation of molecular imaging of thrombosis.

\section{DISCLOSURE}

The costs of publication of this article were defrayed in part by the payment of page charges. Therefore, and solely to indicate this fact, this article is hereby marked "advertisement" in accordance with 18 USC section 1734. This work was supported by HL109448 from the National Heart, Lung, and Blood Institute. The smallanimal PET/SPECT/CT system was funded by RR029495 from the National Center for Research Resources. Peter Caravan has equity in Factor 1A, LLC, the company holding the patent rights to the peptide used in this work. No other potential conflict of interest relevant to this article was reported.

\section{REFERENCES}

1. Go AS, Mozaffarian D, Roger VL, et al. Heart disease and stroke statistics: 2013 update-a report from the American Heart Association. Circulation. 2013;127: e6-e245.

2. Omran H, Jung W, Rabahieh R, et al. Imaging of thrombi and assessment of left atrial appendage function: a prospective study comparing transthoracic and transoesophageal echocardiography. Heart. 1999;81:192-198.

3. Zierler BK. Ultrasonography and diagnosis of venous thromboembolism. Circulation. 2004;109(12, suppl 1):19-14.

4. Fedullo PF, Tapson V. Clinical practice: the evaluation of suspected pulmonary embolism. N Engl J Med. 2003;349:1247-1256.

5. Jaffer FA, Tung CH, Wykrzykowska JJ, et al. Molecular imaging of factor XIIIa activity in thrombosis using a novel, near-infrared fluorescent contrast agent that covalently links to thrombi. Circulation. 2004;110:170-176.

6. Wang X, Hagemeyer C, Hohmann J, et al. Novel single-chain antibody-targeted microbubbles for molecular ultrasound imaging of thrombosis: validation of a unique noninvasive method for rapid and sensitive detection of thrombi and monitoring of success or failure of thrombolysis in mice. Circulation. 2012;125: 3117-3126.

7. Botnar RM, Perez AS, Witte S, et al. In vivo molecular imaging of acute and subacute thrombosis using a fibrin-binding magnetic resonance imaging contrast agent. Circulation. 2004;109:2023-2029.

8. Morris TA, Gerometta M, Yusen R, et al. Detection of pulmonary emboli with ${ }^{99 m}$ Tc-labeled anti-D-dimer (DI-80B3)Fab' fragments (ThromboView). Am J Respir Crit Care Med. 2011;184:708-714. 
9. Ciesienski KL, Caravan P. Molecular MRI of thrombosis. Curr Cardiovasc Imaging Rep. 2010;4:77-84.

10. Uppal R, Ay I, Dai G, Kim YR, Sorensen AG, Caravan P. Molecular MRI of intracranial thrombus in a rat ischemic stroke model. Stroke. 2010;41:12711277 .

11. Vymazal J, Spuentrup E, Cardenas-Molina G, et al. Thrombus imaging with fibrin-specific gadolinium-based MR contrast agent EP-2104R: results of a phase II clinical study of feasibility. Invest Radiol. 2009;44:697-704.

12. Uppal R, Catana C, Ay I, Benner T, Sorensen AG, Caravan P. Bimodal thrombus imaging: simultaneous PET/MR imaging with a fibrin-targeted dual PET/MR probe-feasibility study in rat model. Radiology. 2011;258:812-820.

13. Ciesienski KL, Yang Y, Ay I, et al. Fibrin-targeted PET probes for the detection of thrombi. Mol Pharm. 2013;10:1100-1110.

14. Wadas TJ, Wong EH, Weisman GR, Anderson CJ. Coordinating radiometals of copper, gallium, indium, yttrium, and zirconium for PET and SPECT imaging of disease. Chem Rev. 2010;110:2858-2902.

15. McBride WJ, Sharkey RM, Karacay H, et al. A novel method of F-18 radiolabeling for PET. J Nucl Med. 2009;50:991-998.

16. Guide for the Care and Use of Laboratory Animals. Bethesda, MD: National Institutes of Health; 1985. NIH publication 85-23.
17. Loening AM, Gambhir SS. AMIDE: a free software tool for multimodality medical image analysis. Mol Imaging. 2003;2:131-137.

18. Olafsen T, Wu A. Antibody vectors for imaging. Semin Nucl Med. 2010;40:167-181.

19. Liu Y, Hu X, Liu H, et al. A comparative study of radiolabeled bombesin analogs for the PET imaging of prostate cancer. J Nucl Med. 2013;54:2132-2138.

20. Varasteh $\mathrm{Z}$, Aberg $\mathrm{O}$, Velikyan $\mathrm{I}$, et al. In vitro and in vivo evaluation of a ${ }^{18} \mathrm{~F}$ labeled high affinity NOTA conjugated bombesin antagonist as a PET ligand for GRPR-targeted tumor imaging. PLoS ONE. 2013;8:e81932.

21. Bini A, Fenoglio J, Sobel J, Owen J, Fejgl M, Kaplan K. Immunochemical characterization of fibrinogen, fibrin I, and fibrin II in human thrombi and atherosclerotic lesions. Blood. 1987;69:1038-1045.

22. Sato Y, Hatakeyama K, Yamashita A, Marutsuka K, Sumiyoshi A, Asada Y. Proportion of fibrin and platelets differs in thrombi on ruptured and eroded coronary atherosclerotic plaques in humans. Heart. 2005;91:526-530.

23. Uppal R, Medarova Z, Farrar CT, Dai G, Moore A, Caravan P. Molecular imaging of fibrin in a breast cancer xenograft mouse model. Invest Radiol. 2012;47:553-558

24. Wan W, Guo N, Pan D, et al. First experience of ${ }^{18} \mathrm{~F}$-alfatide in lung cancer patients using a new lyophilized kit for rapid radiofluorination. J Nucl Med. 2013;54:691-698. 